\title{
Numerical Design Methodology for an All Superconducting Linear Synchronous Motor
}

\author{
João Murta Pina ${ }^{1}$, Mário Ventim Neves ${ }^{1}$, Alfredo Álvarez ${ }^{2}$, \\ and Amadeu Leão Rodrigues ${ }^{1}$ \\ ${ }^{1}$ Centre of Technology and Systems, \\ Faculdade de Ciências e Tecnologia, Universidade Nova de Lisboa, \\ Monte de Caparica, 2829-516 Caparica, Portugal \\ jmmp@fct.unl.pt, ventim@uninova.pt, leao@uninova.pt \\ 2 "Benito Mahedero" Group of Electrical Applications of Superconductors, \\ Escuela de Ingenierías Industriales, University of Extremadura \\ Avenida de Elvas s/n, 06006 Badajoz, Spain \\ aalvarez@unex.es
}

\begin{abstract}
One potential advantage of the application of superconducting materials in electrical machines is the possibility to build lighter and compact devices by removing iron. These machines find applications, e.g., in systems where cryogenics is already available, or in naturally cryogenic environments. The design of motors with high temperature superconductors (HTS) presents issues unconsidered in classical machines, besides considerations on cryogenics, such as HTS brittleness or mechanical restrictions. Moreover, HTS' electromagnetic properties also degrade due to flux density components, which arise if there is no iron to guide magnetic flux. Several aspects must thus be considered in the design stage, as applications may turn less attractive or even unfeasible. In this paper these issues are detailed, and a numerical methodology for the design of an all superconducting (without iron or conventional conductors) linear synchronous motor is presented.
\end{abstract}

Keywords: High temperature superconductivity, ironless motor, linear synchronous motor.

\section{Introduction}

The motives underlying the use of high temperature superconducting (HTS) materials in electrical motors are guided by their particular features that make them, in certain applications, advantageous when comparing to devices with conventional conductors and/or permanent magnets. Amongst these characteristics highlights the transport of high currents with minimum losses (up to $10^{4} \mathrm{~A} / \mathrm{cm}^{2}$ at $77 \mathrm{~K}$ and $5 \mathrm{~T}$, in second generation HTS tapes [1]) when compared to copper or aluminum conductors, the consequent generation of flux densities equivalently high, and the phenomenon of magnetic flux pinning. It is thus sometimes possible to remove iron from the devices, allowing for lighter and compact machines. HTS machines find potential application in environments where cryogenics is already available, as power industry [2-4] or 
induction heating plants [5], or in naturally cryogenic environments, as future scientific stations in double-shaded craters near Moon's poles [6], where temperature does not rise above $50 \mathrm{~K}$. The design of superconducting motors shows several specifications that do not arise in classical motors' design, besides considerations on cryogenics, such as HTS tapes bending limitations when these are used in armatures' windings. Besides, its electromagnetic properties also degrade due to the presence of flux density components perpendicular to tape surface, when there is no iron to guide magnetic flux. Consequently, applications may turn less attractive or even unfeasible.

Several types of HTS machines have been built in the past, as homopolar [7], synchronous [8-9], reluctance [10], hysteresis [11] and linear motors. Regarding the latter, HTS have been considered either in the armature [12] or excitation field [13].

This paper's main goal is to examine the key issues in the design of an all superconducting linear synchronous motor with HTS both in the armature and in the excitation system. The motor has no ferromagnetic materials or conventional electrical conductors, thus the designation "all superconducting". The final achievement is a numerical methodology for this type of motor design, incomparably faster than tools as e.g. finite elements software.

Next section examines this work's contribution to technological innovation. After that, the motor's architecture is described, followed by the description of the numerical methodology proposed for the determination of forces. Experimental results are shown in the sequel and conclusions are drawn in the end.

\section{Contribution to Sustainability}

HTS materials are foreseen as vehicles of important developments in the Energy field, allowing the advent of new technologies that would be unfeasible or even impossible with other approaches. Amongst some of the (many) current sustainability issues there is energy distribution in dense urban areas, integration of distributed generation in existing grids, or specific requirements to obtain lighter and compact electrical machines. For each of these problems, superconductivity has one or several answers, although commercial applications are still hard to find. Some factors contributing to this evidence are, besides the degree of reliability of current technologies, the need for cryogenics - whose trivialization depends too on the advent of HTS technologies but also on the unavailability of practical and efficient design tools for HTS devices. This work intends to contribute to the latter issue, by presenting a methodology for aided design of HTS machines. One of this work's main achievements is that it allows replacing time consuming design tools like finite elements software, thus improving HTS technologies development, potential enablers of sustainable energy use.

\section{Motor's Architecture}

Ferromagnetic materials are used in electrical machines as guiders and amplifiers of magnetic flux. However, these materials impose restrictions in machines design: one related with magnetic induction saturation (typically bellow $1.8 \mathrm{~T}$ [14]), which leads to nonlinearities and complicates design; the other related with materials physical properties, namely its high density, that makes machines' weight and size determinant for high specific powers [15]. Moreover, hysteresis losses are generated in iron. These motivates the design of ironless motors, by the use of HTS materials. 


\subsection{Armature}

The armature is built by single layer HTS tape $\mathrm{Bi}-2223\left(\mathrm{Bi}_{2} \mathrm{Sr}_{2} \mathrm{Ca}_{2} \mathrm{Cu}_{3} \mathrm{O}_{14}\right)$, with $90 \mathrm{~A}$ critical current, corresponding to $93 \mathrm{~A} / \mathrm{mm}^{2}$ engineering critical current density. This is one order higher than copper current density considered in machines design, about $4 \mathrm{~A} / \mathrm{mm}^{2}$ [14]. One armature's coil is represented in Fig. 1, and its parameters are described in Table 1. The windings were manufactured using previously machined nylon moulds. According to winding dimensions, the pole pitch, $\tau$, is given by

$$
\tau=3\left(l_{w}+g\right)=219 \mathrm{~mm} .
$$

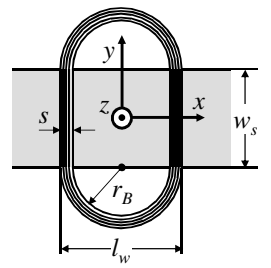

Fig. 1. Representation of one armature winding, build by Bi-2223 stacked tape

Table 1. Armature winding characteristics

\begin{tabular}{lll}
\hline Variable & Meaning & Value \\
\hline$N$ & Number of turns & 20 \\
$s$ & Average coil's leg width & $5 \mathrm{~mm}$ \\
$r_{b}$ & Bending radius & $30 \mathrm{~mm}$ \\
$l_{w}=2 \times\left(r_{b}+s\right)$ & Average coils width & $70 \mathrm{~mm}$ \\
$h_{s}$ & Coils height & $4.2 \mathrm{~mm}$ \\
$g$ & Average distance between adjacent coils & $3 \mathrm{~mm}$ \\
$w_{s}$ & Active coils' length & $80 \mathrm{~mm}$ \\
\hline
\end{tabular}

The armature is built by a double stator, in order to minimize flux density components perpendicular to tape surface, i.e. in $x$ direction, as was the case in this motor's previous geometries [16-18]. These components severely degrade tape's critical current.

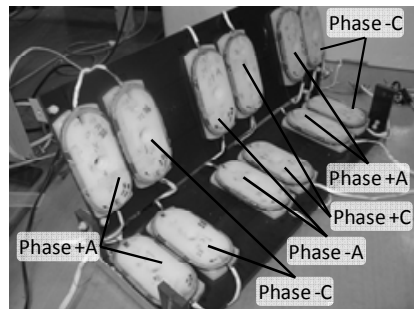

Fig. 2. Test armature built by a three-phase double stator, assembled for experimental measurements. Phase B is not implemented as is not necessary for static measurements, as later discussed. 
A three-phase double stator test armature was built in order to make flux density measurements, see Fig. 2. Only phases A and B are shown, as later explained. Structural fastenings are built with nylon screws to avoid magnetic fields' distortions. In order to determine the developed static forces, the armature is considered to be fed by an ideal current inverter, see currents' profiles in Fig. 3.

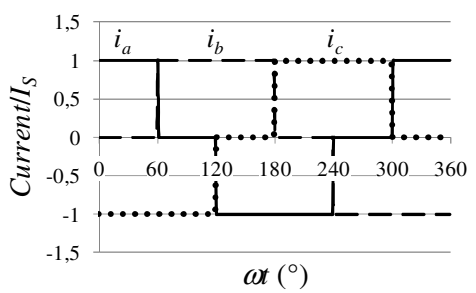

Fig. 3. Normalized armature's three-phase currents with amplitude $I_{S}$, generated by an ideal current inverter. $i_{a}, i_{b}$ and $i_{c}$ are currents from phases $\mathrm{A}, \mathrm{B}$ and $\mathrm{C}$, respectively.

\subsection{Mover}

The mover, comprising excitation, is built by two HTS Y-123 $\left(\mathrm{Y}_{1.6} \mathrm{Ba}_{2} \mathrm{Cu}_{3} \mathrm{O}_{7-x}\right)$ bulks, with, see Fig. 4, magnetized prior to motor's operation. Sand-pile model [19] is used in order to numerically determine trapped flux, considering constant current according to Bean's model [20]. These already demonstrated results consistent with experiments [21]. Considering a $5.2507800 \mathrm{kA} / \mathrm{cm}^{2}$ critical current, see later, and single domain bulks, the computed components of trapped flux, in a plane at $2 \mathrm{~mm}$ from bulk's surface, are shown in Fig. 5. The mover is represented in Fig. 6.

\section{Numerical Determination of Developed Forces}

In order to calculate the developed forces due to the interaction of armature currents with amplitude $I_{S}$ and trapped fields, Laplace's law is applied to excitation,

$$
d \vec{F}=-I_{S} d \vec{l} \times \vec{B}
$$

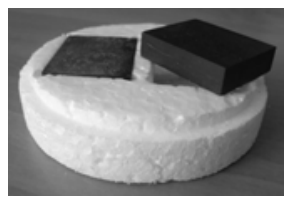

Fig. 4. Y-123 bulks (ATZ GmbH), used as trapped flux magnets for motor's excitation $\left(40 \times 32 \times 10 \mathrm{~mm}^{3}\right)$ 

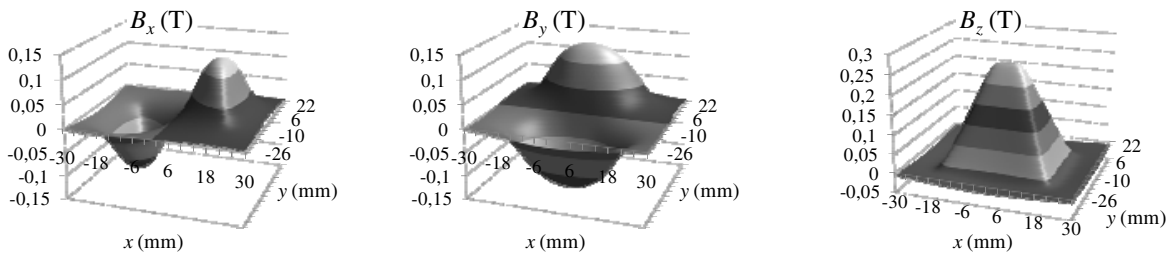

Fig. 5. Flux density components in an Y-123 bulk, numerically determined by sand-pile and Bean models, measured at $2 \mathrm{~mm}$ from bulk surface, and a critical current of $5.2507800 \mathrm{kA} / \mathrm{cm}^{2}$

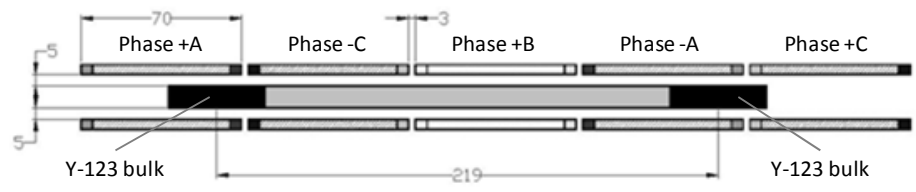

Fig. 6. Representation of the linear motor's mover between armature windings. All dimensions in millimeters.

This equation is integrated along the height and width of armature windings. According to the coordinate system defined in Fig. 1, and considering $d \vec{l}=-d y \vec{u}_{y}$, the several force components are derived, namely

$$
\begin{gathered}
\text { Thrust force: } d F_{x}=I_{S} B_{z} d y \\
\text { Vertical force: } d F_{z}=-I_{S} B_{x} d y \\
\text { Lateral force: } d F_{y}=0 .
\end{gathered}
$$

Thus, $B_{y}$ components do not contribute to developed forces, as they are parallel to armature current in the active region. First, the forces produced by a half stator are computed, and in the end the other half stator's contribution is added. Since flux density changes across windings height, its values are first averaged over $z$ dimension, thus defining the following functions

$$
\begin{aligned}
& B_{x}^{a v}(x, y)=\frac{1}{h_{s}} \int_{h_{s}} B_{x}(x, y, z) d z \\
& B_{z}^{a v}(x, y)=\frac{1}{h_{s}} \int_{h_{s}} B_{z}(x, y, z) d z .
\end{aligned}
$$

These functions are plotted in Fig. 7. After that, (6) and (7) must be averaged across winding's active length, thus originating functions

$$
\begin{aligned}
& B_{x y}^{a v}(x)=\frac{1}{w_{s}} \int_{w_{s}} B_{x}^{a v}(x, y) d y \\
& B_{z y}^{a v}(x)=\frac{1}{h_{s}} \int_{h_{s}} B_{z}^{a v}(x, y) d y,
\end{aligned}
$$


which are plotted in Fig. 8. Functions $B_{x y}^{a v}$ and $B_{z y}^{a v}$ must contain the two Y-123 bulks, magnetized in opposite directions. The complete functions are plotted in Fig. 9.
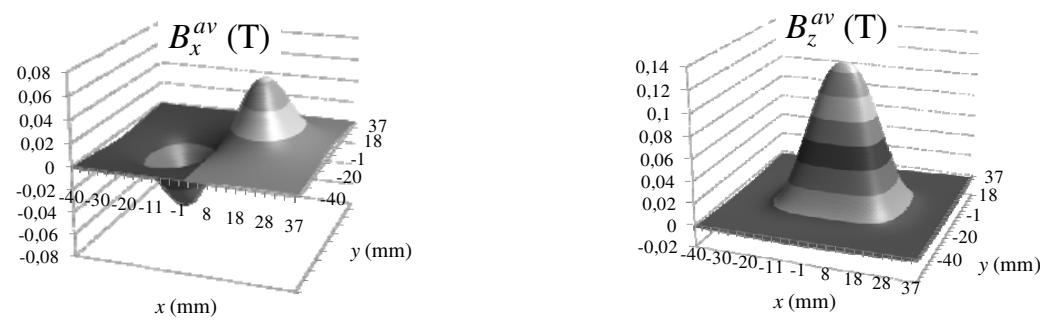

Fig. 7. Flux density average of one Y-123 trapped flux bulk along winding's height

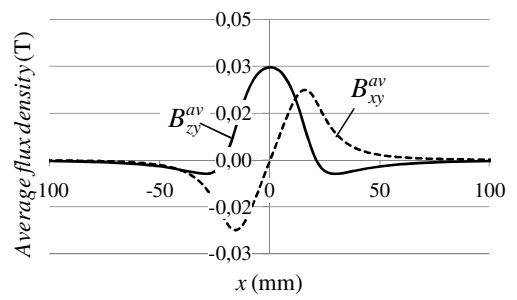

Fig. 8. Flux density average of one Y-123 trapped flux bulk along winding's height and length

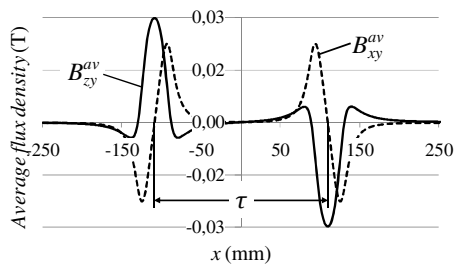

Fig. 9. Flux density average of the two Y-123 trapped flux bulks along winding's height and active length

The mover's position is varied from $x=0$ to $2 \tau$, (or from $\theta=0^{\circ}$ to $360^{\circ}$, where $\theta=180^{\circ} x / \tau$ is the angular displacement). In each position, forces are calculated by

$$
\begin{aligned}
& F_{x}(\theta)=\int_{-\infty}^{\infty} c(x) B_{z y}^{a v}\left(x-\tau \frac{\theta}{180^{\circ}}\right) d x \\
& F_{z}(\theta)=\int_{-\infty}^{\infty} c(x) B_{x y}^{a v}\left(x-\tau \frac{\theta}{180^{\circ}}\right) d x .
\end{aligned}
$$


In (10) and (11), $c(x)$ represents current elements along armature. This is plotted in Fig. 10 as a function of $\theta$, taking values $\pm I_{S} N / s$ where there is current density and zero in the other regions. For static forces' calculation a time instant in range $\omega t \in] 0^{\circ}, 60^{\circ}\left[\right.$ is chosen, corresponding to $i_{a}=I_{S}, i_{b}=0$ and $i_{c}=-I_{S}$, with $I_{S}=65 \mathrm{~A}$, see Fig. 3. This is why phase B is not implemented. Equations (6) to (11) are numerically integrated, returning thrust and vertical static forces corresponding to only one half stator. The other half's contribution means that that thrust is doubled, and vertical force is subtracted from itself, thus resulting in zero for all positions. Thrust force is represented in Fig. 11. It is clear that thrust force is highly oscillating, which is a consequence of the high space harmonics' content of magnetomotive force, due to Bi-2223 windings. This creates control issues outside the scope of this paper.

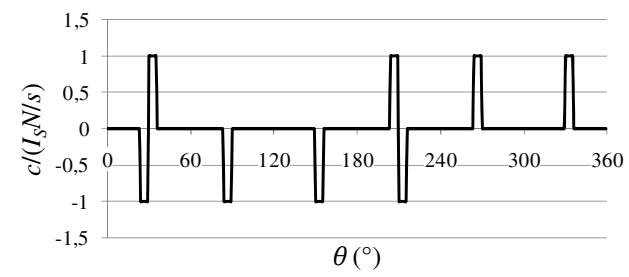

Fig. 10. Function $c(\theta)$ describing currents' profile along armature, for $\omega t \in] 0^{\circ}, 60^{\circ}[$

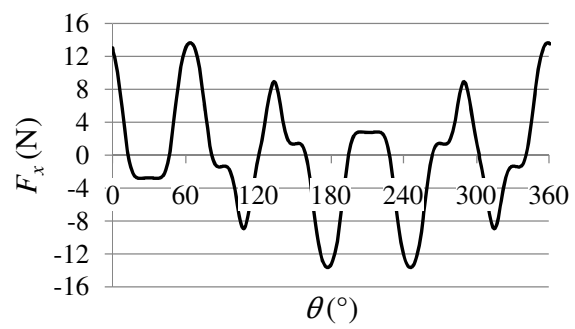

Fig. 11. Thrust force developed on the mover, as a consequence of the two half stators

\subsection{Measurement of Flux Density Generated by the Armature}

To measure flux density profile, a transversal flux Hall probe assembled in a $x y z$ positioner was used, phases $\mathrm{A}$ and $\mathrm{C}$ fed by, respectively, 65 and $-65 \mathrm{~A}$, and phase $\mathrm{B}$ set to zero. The profile along a longitudinal path is plotted in Fig. 12, as well as the consistent results obtained by simulation with finite elements software Flux2D.

\subsection{Measurement of Trapped Flux Density}

In order to magnetize Y-123 bulks, four 400 A DC current sources in parallel were used. The bulk is placed in the middle of two air core inductors, supplied by a $1000 \mathrm{~A}$ current peak from the sources. Magnetic flux gets trapped in the bulk's pinning 
centers, magnetizing it. A transversal Hall probe assembled on the same positioner was then used to measure trapped flux profile, shown in Fig. 13. Maximum flux density is $209 \mathrm{mT}$, which at $77 \mathrm{~K}$ cannot be increased, as the bulk is fully penetrated. By fitting curves obtained with sand-pile and Bean models, considering one single domain, it is possible to estimate a current density of $5,2507800 \mathrm{kA} / \mathrm{cm}^{2}$. This is the value used in thrust force determination. The modeled profile is shown in Fig. 14.

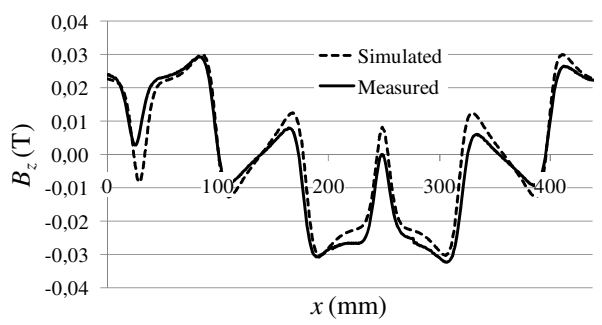

Fig. 12. Flux density component $B_{z}$ measured along a longitudinal path across the armature, at middle distance between the two half stators. Simulated field is also shown for comparison.

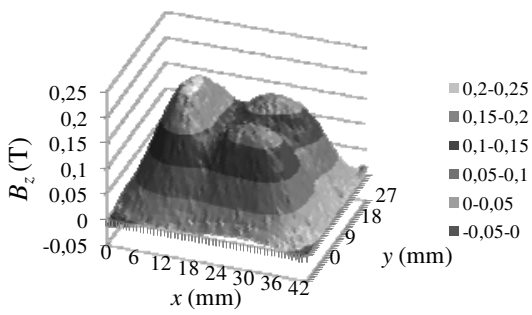

Fig. 13. Flux trapped in one bulk, after a current pulse. The peak from the left corresponds to one domain, while the two other peaks correspond to the other domain, which is damaged.

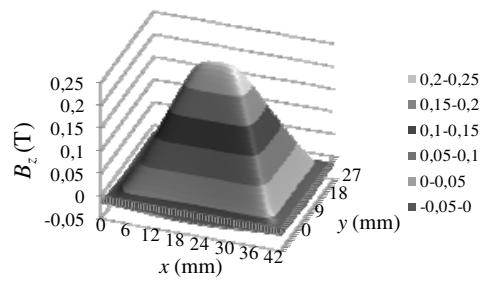

Fig. 14. Modeling of flux trapped in one Y-123 bulk, by sand-pile and Bean models

\section{Conclusions and Future Work}

An all superconducting linear synchronous motor was presented in this paper, as well as the methodology to derive the forces developed by this device. The main advantage of this motor is its reduced weight when compared with conventional motors, due to the absence of iron as well as copper conductors. This work's main goal, which is the 
development of a numerical methodology that allows avoiding time consuming finite elements software for HTS devices design, is accomplished. Future work includes finishing the motor, validate numerical results, and compare its performance when iron is included.

Acknowledgments. This work was supported by FCT (CTS multiannual funding) through the PIDDAC Program funds.

\section{References}

1. Lee, P.J. (ed.): Engineering Superconductivity, pp. 260-280. Wiley-IEEE Press (2001)

2. Maguire, J.F., Yuan, J.: Status of high temperature superconductor cable and fault current limiter projects at American Superconductor. Physica C 469(15-20), 874-880 (2009)

3. Reis, C.T., Mehta, S.P., McConnell, B.W., Jones, R.H.: Development of High Temperature Superconducting Power Transformers. In: Power Engineering Society Winter Meeting 2001, pp. 432-437. IEEE, Ohio (2001)

4. Paul, W., Lakner, M., Rhyner, J., Unternährer, P., Baumann, T., et al.: Test of 1.2 MVA high-Tc superconducting fault current limiter. Superc. Sci. Tech. 10(12), 914-918 (1997)

5. Morandi, A., Fabbri, M., Ribani, P.L.: Design of a Superconducting Saddle Magnet for DC Induction Heating of Aluminum Billets. IEEE Trans. Appl. Superc. 18(2), 816-819 (2008)

6. Ryan, R.E., Underwood, L.W., McKellip, R., Brannon, D.P., Russel, K.J.: Exploiting Lunar Natural and Augmented Thermal Environments for Exploration and Research. In: 39th Lunar and Planetary Science Conference, Texas (2008)

7. Superczynski, M.J., Waltman, D.J.: Homopolar Motor with High Temperature Superconductor Field Windings. IEEE Trans. Appl. Superc. 7(2), 513-518 (1997)

8. Frank, M., van Hasselt, P., Kummeth, P., Massek, P., Nick, W., et al.: High-Temperature Superconducting Rotating Machines for Ship Applications. IEEE Trans. Appl. Superc. 16(2), 1465-1468 (2006)

9. Buck, J., Hartman, B., Ricket, R., Gamble, B., MacDonald, T., Snitchler, G.: Factory Testing of a 36.5 MW High Temperature Superconducting Propulsion Motor. In: Fuel Tank to Target: Building the Electric Fighting Ship at American Society of Naval Engineers Day 2007, Arlington (2007)

10. Oswald, B., Best, K.-J., Setzer, M., Soll, M., Gawalek, W., et al.: Reluctance Motors with Bulk HTS Material. Superc. Sci. Tech. 18, 24-29 (2005)

11. Kovalev, L.K., Ilushin, K.V., Penkin, V.T., Kovalev, K.L., Koneev, S.M.-A., et al.: Hysteresis and Reluctance Electric Machines with Bulk HTS Elements. Recent Results and Future Development. Superc. Sci. Tech. 13(5), 498-502 (2002)

12. Kim, W.-S., Jung, S.-Y., Choi, H.-Y., Jung, H.-K., Kim, J.H., Hahn, S.-Y.: Development of a Superconducting Linear Synchronous Motor. IEEE Trans. Appl. Superc. 12(1), 842-845 (2002)

13. Sato, A., Ueda, H., Ishiyama, A.: Operational Characteristic of Linear Synchronous Actuator with Field-Cooled HTS Bulk Secondary. IEEE Trans. Appl. Superc. 15(2), 2234-2237 (2005)

14. Say, M.G.: The Performance and Design of Alternating Current Machines: Transformers, Three-Phase Induction Motors and Synchronous Machines, 3rd edn. CBS Publishers \& Distributers, New Delhi (1983) 
15. Vajda, I., Szalay, A., Gobl, N., Meerovich, V., Sokolovsky, V.: Requirements for the industrial application of superconducting rotating electrical machines. IEEE Trans. Appl. Superc. 9(2), 1225-1228 (1999)

16. Pina, J.M., Ventim Neves, M., McCulloch, M.D., Rodrigues, A.L.: Design of a linear synchronous motor with high temperature superconductor materials in the armature and in the field excitacion system. J. Phys: Conf. Series, 43(1), 804-808 (2006)

17. Pina, J.M., Ventim Neves, M., Rodrigues, A.L.: Case Study in the Design of HTS Machines: an All Superconducting Linear Synchronous Motor. In: International Conference on Power Engineering, Energy and Electrical Drives, POWERENG 2007, Setúbal, pp. 185-190 (2007)

18. Pina, J., Gonçalves, A., Pereira, P., Álvarez, A., Ventim Neves, M., Rodrigues, A.: A test rig for thrust force measurement of an all HTS linear synchronous motor. J. Phys: Conf. Series, 97(1), 12220 (2008)

19. Fukai, H., Tomita, M., Murakami, M., Nagatomo, T.: Numerical simulation of trapped magnetic field for bulk superconductor. Physica C 357-360, Part 1, 774-776 (2001)

20. Bean, C.P.: Magnetization of High-Field Superconductors. Rev. Mod. Phys. 36, 31-39 (1964)

21. Aydiner, A., Yanmaz, E.: Numerical calculation of trapped magnetic field for square and cylindrical superconductors. Superc. Sci. Tech. 18(7), 1010-1015 (2005) 